\title{
Propuesta metodológica para incorporar la creación del conocimiento a los sistemas de gestión en las entidades del Estado del nivel nacional ${ }^{*}$
}

\section{Methodological purpose to incorporate the knowledge creation into management systems in Government institutions}

Recibido: 4 de junio de 2015 Revisado: 14 de agosto de 2015 Aceptado: 19 de octubre de 2015

Carolina Orjuela Russi*

Policía Nacional de Colombia

Rosario del Pilar Ramos Día:

Universidad Santo Tomás

Gloria Matilde Torres Cruz***

Convenio USTA - Icontec

\section{RESUMEN}

ElEstado colombiano definiólos sistemas de gestión de Modelo Estándar de Control Interno. Posteriormente, calidad y control interno como herramientas de gestión en pro del mejoramiento continuo de la administración de obligatoria implementación bajo los referentes de la pública y de la mejora de la gestión, se especificaron Norma Técnica de Calidad en la Gestión Pública y el lineamientos adicionales asociados a las políticas de

* Artículo de resultado de investigación: "Propuesta metodológica para incorporar la creación del conocimiento a los sistemas de gestión en las entidades del Estado a nivel nacional", 2015.

** Administradora de Empresas. Especialista en Finanzas. Magíster en Calidad y Gestión Integral. Policía Nacional de Colombia. Correo electrónico: carolina.orjuela1206@correo.policia.gov.co

*** Ingeniera Industrial. Magíster en Calidad y Gestión Integral. Correo electrónico: torrescruzg@gmail.com 
desarrollo administrativo, los cuales generaron en las entidades públicas la incorporación de requerimientos que impactan estos sistemas produciendo desgastes administrativos, fallas en el control de los procesos, desinformación y sobrecostos, entre otros. Este proyecto de investigación buscó crear una propuesta metodológica que permita a los entes gubernamentales nacionales incorporar algunos conceptos como políticas, requerimientos, temas o componentes (entre otros significados) a sus modelos de gestión de calidad y normas de control interno realizados previamente y basados en esos modelos. Por lo anterior, analizada la Teoría de Creación del Conocimiento Organizacional de Nonaka y Kakeuchi, frente a los sistemas de gestión de calidad y control interno aplicables a las entidades del Estado colombiano del ámbito nacional, se identificó un vínculo que reconoce la existencia de condiciones que facilitan el flujo del conocimiento a través de la socialización entre individuos y organizaciones, toda vez que se establecen lineamientos que propician, regulan, justifican y promueven la generación de nuevos conceptos, para incorporarlos a la gestión institucional. Definida la propuesta, se realizó un ejercicio de validación en la ANLA para incorporar el objeto de conocimiento "participación ciudadana y control social" a sus sistemas y se estableció que la articulación de las fases de creación del conocimiento organizacional con los sistemas de gestión, facilita la incorporación de nuevos objetos de conocimiento garantizando la integridad de los sistemas ya implementados.

Palabras clave: gestión del conocimiento, sistemas de gestión, entidades públicas, NTCGP 1000, MECI.

\section{ABSTRACT}

Colombian Government established the quality management and internal control as mandatory tools of management, according to Quality Technical Standards
NTCGP:1000 and the Standard Model of Internal Control, incorporated by government in decrees 4485 of 2009 and 1599 of 2005 respectively. Moreover, looking for the continuous improvement of public administration and the capability to enhance its arrangement, Colombian government has defined another kind of policies and organizational methodologies mainly related to the administrative development, which has induced to national government entities to incorporate the requirements that affect among others implemented quality systems, involving administrative wastage, out of control over processes, not enough information and overcosts. Current research project tries to create a methodological purpose that let national government entities, incorporate some concepts as policies, requirements, topics or components (among other meanings) to its models of quality management and internal control standards accomplished previously and based on those models are a complement that must be adopted. Therefore and once analized the Theory of Organizational Knowledge Creation raised by Nonaka and Takeuchi in their book "The Knowledge Creating Company", the quality management systems and internal control standards applied to national government entities, there is an identified linkage that recognizes that some conditions help to the knowledge flow, between people, groups and organizations, due that policies bring on, adjust, control and promote the new concepts generation, getting its incorporation to institutional management. Once the methodological purpose is defined, there was a validation test in National Authority of Environmental Licenses Agency, in order to incorporate the knowledge object named "Citizen participation and social control" to the implemented systems, that let establish and link up the creation phases of organizational knowledge to the quality management systems and internal control, so incorporate new knowledge objects assure wholeness to implemented systems in an easier way. 
Keywords: Knowledge, organizational knowledge creation, management systems, government institutions, spiral of knowledge.

\section{INTRODUCCIÓN}

El Estado colombiano buscando la excelencia de las entidades, para ello ha definido herramientas de gestión como la Norma Técnica de Calidad en la Gestión Pública - NTCGP 1000:2009 (Icontec, 2009) y del Modelo Estándar de Control Interno - MECI 1000:2014, cuyas concepciones están basadas en la Norma Técnica de Calidad ISO y la metodología COSO (Committee of Sponsoring Organizations) respectivamente, siendo estas de obligatorio cumplimiento; sin embargo, se han venido sumando otro tipo de políticas, estrategias e iniciativas de gobierno en su mayor parte asociadas a las Políticas de Desarrollo Administrativo (Congreso de la República, Ley 489, 1998), cuya implementación gira en torno a la gestión misional y de gobierno, la transparencia, la participación y servicio al ciudadano, la gestión del talento humano, la eficiencia administrativa y la gestión financiera.

Producto de esta situación, las entidades del Estado implementan los lineamientos gubernamentales de manera desarticulada a los sistemas de gestión de calidad y de control interno, generando, entre otros, desfragmentación de las estrategias y procesos implementados.

Partiendo de este contexto, es relevante determinar una propuesta metodológica que permita a las entidades del Estado del nivel nacional la incorporación de conceptos tales como lineamientos, requisitos, temas o componentes (entre otras denominaciones) a sus modelos de gestión de calidad y de control interno previamente implementados, fundamentada en que dichos modelos son complementarios y de obligatoria adopción.
Por consiguiente, y con el fin de desarrollar la propuesta metodológica, se toma como fundamento las ideas planteadas por los autores Nonaka y Takeuchi (2009): "Toda vez que se valora la existencia de conocimiento previo dentro de la entidad, frente a cualquier objeto de conocimiento susceptible de incorporar a la gestión institucional, a través de la información que poseen los individuos que la conforman" (p. 64). Lo que permite la definición de nuevos conceptos que una vez son identificados, se implementan en la organización de forma articulada a los sistemas existentes, conservando la integridad de los mismos.

Por lo anterior, se establece una relación entre las fases de la creación del conocimiento organizacional propuestas por Nonaka y Takeuchi con los principios, requisitos y elementos definidos en los modelos de gestión de calidad y de control interno. Lo que permitió la consolidación de la propuesta metodológica, la cual fue validada mediante la realización de un ejercicio práctico en la Autoridad Nacional de Licencias Ambientales (ANLA) buscando la incorporación del concepto "participación ciudadana y control social".

\section{METODOLOGÍA}

La investigación se fundamenta en el pragmatismo, que involucra una multiplicidad de perspectivas, premisas teoréticas, tradiciones metodológicas, técnicas de recolección, análisis de datos, entendimientos y valores que constituyen los elementos de los modelos mentales (Hernández, Fernández y Baptista, 2010, p. 553). Toda vez que al revisar la Teoría de Creación del Conocimiento Organizacional y los sistemas de gestión de calidad y de control interno con fines específicos, se determinan los elementos comunes sin desconocer la esencia de cada uno de ellos, realizando aportes en pro del desarrollo de un todo. 
Se desarrolló un estudio mixto de tipo exploratorio secuencial, toda vez que inicia con la recolección y análisis de datos cuantitativos, frente a los principios, requisitos y elementos de los modelos de gestión de calidad y de control interno y la creación del conocimiento organizacional planteada por Nonaka y Takeuchi. Lo que permitió desde el análisis cualitativo determinar la integración de los objetos estudiados en una propuesta metodológica, para la incorporación de nuevos elementos de conocimiento a los sistemas de gestión establecidos en las entidades del Estado.

Continuando con la metodología utilizada para lograr la propuesta metodológica, en la etapa de validación se realizó una encuesta estructurada, para determinar el diagnóstico del objeto de conocimiento seleccionado "participación ciudadana y control social" en la Autoridad Nacional de Licencias Ambientales (ANLA).

Por consiguiente, la investigación utilizó el método inductivo a través del proceso de recolección (observación), tabulación (registro), caracterización (clasificación) y estudio de los hechos identificados, para proyectar la definición de preguntas orientadoras (para articular a los principios, requisitos y elementos de los sistemas de gestión de calidad y MECI con las de creación del conocimiento organizacional), y lemas frente a la "participación ciudadana y el control social", para la creación de los nuevos conceptos a incorporar en la Autoridad de Licencias Ambientales (ANLA).

Una vez abordado el fundamento metodológico de la investigación, se establece la validez y confiabilidad de la misma, haciendo revisión de los criterios propuestos por Hernández, Fernández y Baptista (2010), en cuanto a la calidad del diseño mixto, dada por la combinación de técnicas cualitativas y cuantitativas; calidad de las inferencias y rigor interpretativo, expresado en la definición de las preguntas orientadoras que permitieron la articulación de la creación del conocimiento organizacional con los principios, requisitos y elementos del SGC y MECI, la interpretación de los resultados y la definición de la incorporación de nuevos conceptos; la transferencia de resultados, teniendo en cuenta que la propuesta metodológica es un documento genérico que puede ser adoptado y aplicado sin importar la misionalidad de la entidad del Estado; y la legitimidad, teniendo en cuenta que es la construcción de una propuesta metodológica, realizado bajo un análisis cualitativo de datos cuantitativos, y la validación realizada del contenido del documento.

Durante la investigación se implementó la encuesta estructurada, la cual fue aplicada de conformidad a la muestra determinada en la tabla 1 .

Tabla 1. Universo y muestra.

\begin{tabular}{|l|c|c|}
\hline \multirow{2}{*}{ Mecanismo } & ANLA & \\
\cline { 2 - 3 } & $\mathrm{N}$ & N=muestra \\
\hline Encuestas estamento directivo & 11 & 11 \\
\hline Encuestas estamento jurídico & 36 & 35 \\
\hline Encuestas estamento técnico & 308 & 229 \\
\hline Encuestas estamento logístico & 35 & 34 \\
\hline
\end{tabular}

Fuente: elaboración propia.

\section{RESULTADOS Y DISCUSIÓN}

Con la revisión documental, a través de redes conceptuales, se diagramaron los aspectos fundamentales de la Teoría de Creación del Conocimiento Organizacional planteada por Nonaka y Takeuchi y la del Ciclo de la Gestión del Conocimiento planteada por Peluffo y Catalán en el Manual de introducción a la gestión del conocimiento y su aplicación al sector público, con el fin de comparar e identificar la línea de trabajo a seguir en la investigación. 
Por consiguiente, se establece que el proyecto no persigue el objetivo de crear un modelo o sistema de gestión adicional en las entidades públicas del Estado colombiano, si no que busca brindar un mecanismo para que los sistemas ya implementados de gestión de calidad y control interno se constituyan en los elementos de la gestión institucional que faciliten la incorporación nuevos objetos de conocimiento, entendidos estos como las temáticas sobre las cuales la organización ha planificado crear e inducir conocimiento institucional de acuerdo con su realidad, sus objetivos estratégicos y sus necesidades, para actuar y tomar decisiones sobre estas, siendo definido para tal fin la utilización de la Teoría de Creación del Conocimiento Organizacional.

Las discusiones que se presentan se basan en el análisis de las fases de creación del conocimiento con el fin de determinar las preguntas orientadoras que permiten la articulación de la Creación del Conocimiento Organizacional planteada por Nonaka y Takeuchi con los principios, requisitos y elementos del SGC y MECI, dejando claro el enfoque utilizado para la construcción de la propuesta metodológica y la posterior validación de la misma.

La propuesta metodológica, se estructura específicamente para abordar la Creación del Conocimiento Organizacional, asociada a los sistemas de gestión de calidad y de control interno, como una herramienta que orienta a la entidad para incorporar nuevos conceptos sin afectar la integridad de sus sistemas de gestión.

En la primera parte, se identifican aspectos fundamentales de la Teoría de Creación del Conocimiento Organizacional planteados por Nonaka y Takeuchi tales como las dimensiones del conocimiento, las formas de conversión del conocimiento, la espiral de conocimiento organizacional, las condiciones facilitadoras del conocimiento y las fases de creación del conocimiento organizacional.
En la segunda parte, se explica el relacionamiento existente entre la Teoría de Creación del Conocimiento Organizacional y los principios, requisitos y elementos de las normas MECI 1000:2014 y NTCGP 1000:2009 a través de la resolución de las preguntas orientadoras.

Para finalizar, en la tercera parte se establecen los lineamientos o consideraciones a tener en cuenta para poder incorporar objetos de conocimiento a los sistemas de gestión de calidad y de control interno a partir de análisis de los principios, requisitos y elementos de los mencionados sistemas.

Una vez determinado el enfoque, la propuesta metodológica se constituye en una herramienta que facilita la incorporación de nuevos conceptos a los sistemas de gestión de calidad y de control interno implementados en las entidades del Estado colombiano.

\section{LA CREACIÓN DEL CONOCIMIENTO}

Durante la exploración documental realizada para la investigación, se identifica como fundamento de las teorías administrativas dos modelos de creación de conocimiento organizacional: 1) el occidental fundamentado en el racionalismo y 2) el oriental basado en el empirismo. Ambos modelos implican una fuerte interacción entre los individuos de una organización y la generación de sinergias para abordar objetivos de eficiencia organizacional como mecanismo para crear ventajas competitivas que expandan las opciones de crecimiento y/o sostenibilidad.

Para lograr la descripción de cada modelo, se encuentra que las autoras Peluffo y Catalán (2002) describen el modelo occidental de la siguiente manera:

El modelo occidental de generación de conocimiento organizacional proviene de la corriente humanista y 
está basado en el aprendizaje que tiene su origen en las preguntas, cuestionamientos, problemas o necesidades de las personas, grupos u organizaciones, las cuales dan lugar a un conjunto de ideas en busca de las respuestas adecuadas. Estas ideas se ponen a prueba y mediante la reflexión se identifican las mejores soluciones, que son el conocimiento nuevo (p. 61).

Por otra parte, el modelo oriental de creación de conocimiento organizacional está basado en las experiencias de los sujetos que conforman las empresas; proviene de conocimientos tácitos y busca la transformación del conocimiento individual en conocimiento explícito colectivo. Sin duda los principales exponentes del modelo oriental de creación de conocimiento organizacional son los autores Ikujiro Nonaka e Hirotaka Takeuchi (1999, p. 65), quienes explicaron la Teoría de Creación del Conocimiento Organizacional a través de su obra La organización creadora del conocimiento: cómo las compañias japonesas crean la dinámica de la innovación.

Por lo antes expuesto y partiendo de la premisa de que los individuos que conforman las organizaciones se encuentran inmersos en el conocimiento y la información, y que por tanto no son elementos aislados (sujeto y objeto), es decir, los objetos de conocimiento están incorporados a los sujetos a través de la experiencia, en la investigación se abordan los aspectos fundamentales del planteamiento realizado por Nonaka y Takeuchi.

\section{DIMENSIONES DEL CONOCIMIENTO}
Para Nonaka y Takeuchi, la creación del conocimiento organizacional se fundamenta en dos dimensiones del conocimiento, así:

- Epistemológica, que trata del conocimiento tácito, que es aquel asociado al individuo, con dos dimensiones en su interior: una técnica (lo que sabe hacer), y una cognitiva (lo que debería ser); y el conocimiento explicito, que es aquel conocimiento codificado que es fácilmente trasferible.

Fiǵura 1. Dimensión epistemológica del conocimiento.

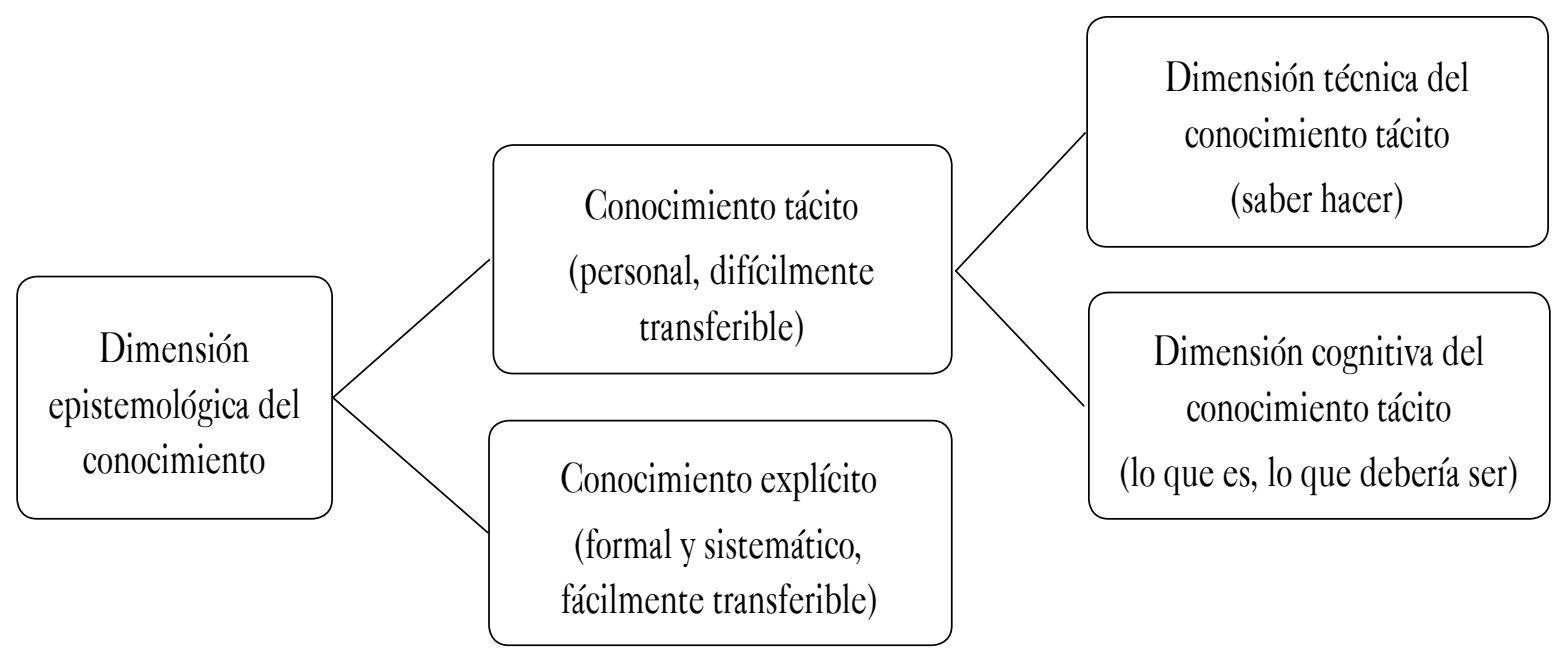

Fuente: elaboración propia a partir de la dimensión epistemológica del conocimiento planteada por Nonaka y Takeuchi en La organización creadora del conocimiento: cómo las compañias japonesas crean la dinámica de la innovación. 
- Ontológica, que habla del conocimiento individual, grupal, organizacional e interorganizacional.

Figura 2. Dimensión ontológica del conocimiento.

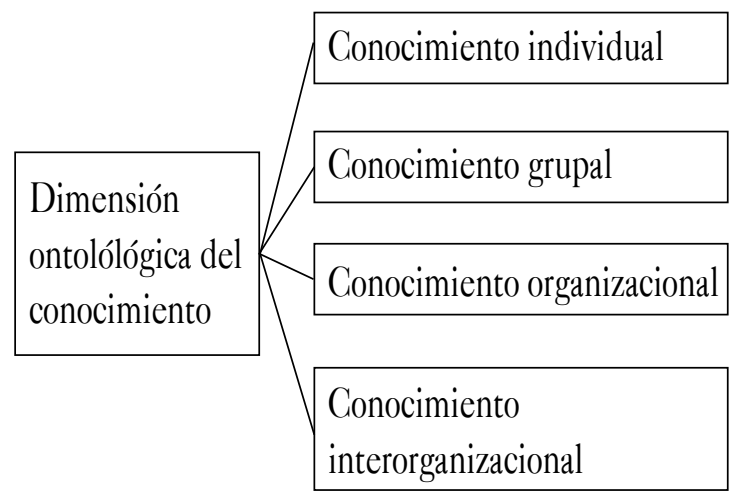

Fuente: elaboración propia a partir de la dimensión ontológica del conocimiento planteada por Nonaka y Takeuchi en La organiæación creadora del conocimiento: cómo las compañias japonesas crean la dinámica de la innovación.

\section{ESPIRAL DE CREACIÓN DEL CONOCIMIENTO}

Para Nonaka y Takeuchi, el conocimiento individual (tácito) se transforma en conocimiento grupal, organizacional e interorganizacional (explícito) a partir del flujo de información. Lo que muestra que la teoría no se concentra en el desarrollo de procesos de enseñanza y aprendizaje, toda vez que pretende es capturar el conocimiento individual y difundirlo en las entidades de manera organizada.

Por lo anterior, Nonaka y Takeuchi (1999, pp. 70-76) plantean cuatro fases de conversión del conocimiento entre los individuos y el colectivo organizacional:

Socialización. Consiste en compartir experiencias y por tanto crear conocimiento tácito, tal como en los modelos mentales compartidos y las habilidades y técnicas. En la socialización la clave para adquirir conocimiento tácito es la experiencia.

Exteriorización. Es el proceso de creación de conceptos generados por el diálogo y la reflexión colectiva. Un método muy utilizado para crear conceptos es combinar la deducción y la inducción.

Combinación. Se da cuando los niveles medios descifran y ponen en operación las visiones corporativas, los conceptos de negocio o los conceptos de producto.

Interiorización. Está relacionada con el aprender haciendo. Se da cuando las experiencias son internalizadas en la base del conocimiento tácito de los individuos a través de la socialización. En esta forma de conversión el conocimiento fácilmente se puede verbalizar o diagramar en documentos, manuales o historias orales, entre otros.

Estas formas de conversión del conocimiento interactúan de manera secuencial y cíclica en el tiempo, haciendo que dicha interacción se comporte como una espiral; es decir, el objeto de conocimiento no es estático, sino que se amplía en la medida que las bases del mismo vuelven a someterse al proceso natural de socialización. A este fenómeno le denominaron la Espiral del Conocimiento Organizacional.

El modelo de Espiral del Conocimiento Organizacional (figura 3) plantea la interacción de las dimensiones epistemológica y ontológica, siendo el vértice el conocimiento tácito o individual. A este modelo bidimensional se le adicionan las cuatro formas de conversión del conocimiento anteriormente tratadas: socialización, exteriorización, combinación e interiorización, formando una espiral que se mueve en un plano tridimensional. 
Figura 3. La Espiral del Conocimiento Organizacional.

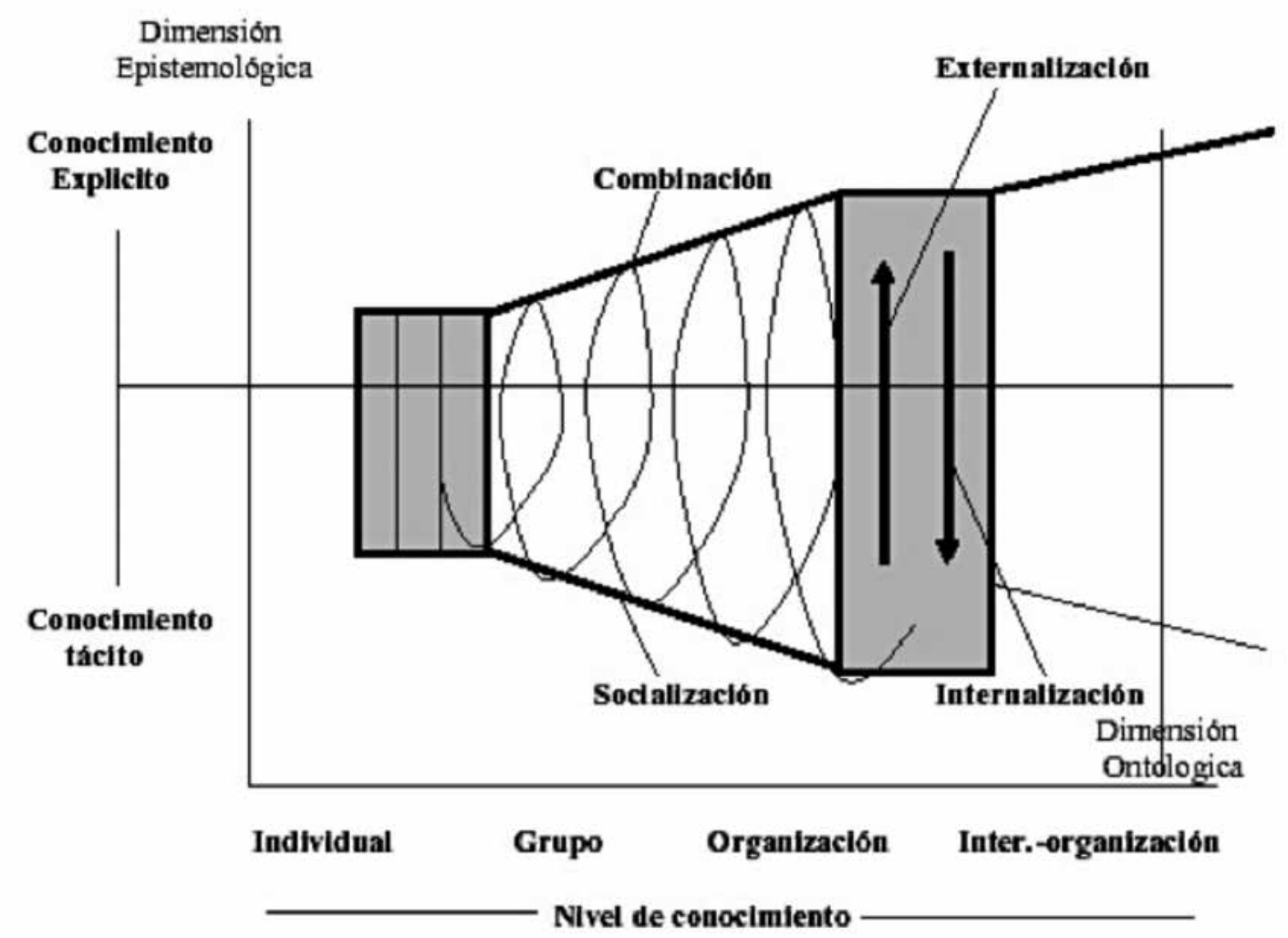

Fuente: Nonaka y Takeuchi (1999). La organización creadora de conocimiento: cómo las compañías japonesas crean la dinámica de la innovación.

El modelo de Espiral del Conocimiento Organizacional se constituye en un referente de cómo las entidades deberían abordar y gestionar el conocimiento. Sin embargo, Nonaka y Takeuchi (1999, pp. 84-91) plantean la existencia de condiciones facilitadoras que contribuyen a mejorar la forma como fluye el conocimiento en la organización, así:

Intención. Corresponde a la aspiración que una empresa tiene por alcanzar sus metas, asumen la forma de una estrategia. La esencia de la estrategia es desarrollar la capacidad organizacional para adquirir, crear, acumular y explotar el conocimiento.

Autonomía. Los individuos y los grupos autónomos establecen los límites de sus tareas por sí mismos, para así perseguir la meta última expresada en la intención total de la organización.

Fluctuación del caos creativo. Consiste en la ruptura de rutinas, hábitos o marcos cognoscitivos. Una ruptura es una interrupción en nuestro estado habitual y cómodo con el fin de reconsiderar pensamientos y perspectivas fundamentales. "Crear conocimiento a partir del caos".

Redundancia. Hace referencia a la sobreexposición intencional de la información acerca de las actividades propias del negocio, de responsabilidades administrativas y de la empresa en su conjunto, es decir el concepto generado por un individuo o un grupo se 
comparte con otros individuos que quizá no necesiten del concepto de manera inmediata.

Variedad de requisitos. Hace referencia a las capacidades de los miembros de la organización. Para fomentar la variedad de requisitos, los autores proponen la disponibilidad indistinta de la información con el fin de que los individuos interactúen en los mismos términos, una estructura orgánica plana y flexible y la rotación del personal con el fin de que se adquiera conocimiento multifuncional.

\section{FASES DE CREACIÓN DEL CONOCIMIENTO}

Una vez expuestas las dimensiones y las formas de conversión del conocimiento, enmarcando las condiciones que fomenta su creación en las organizaciones, los autores proponen cinco fases secuenciales que delimitan la temporalidad de su generación así:

Compartir el conocimiento tácito. Consiste en el intercambio de conocimiento individual.

Crear conceptos. El modelo mental tácito compartido se traduce en palabras, frases y se materializa en conceptos explícitos.

Justificar conceptos. Incluye determinar si los conceptos creados son en verdad válidos para la organización.

Construir un arquetipo. El concepto justificado se convierte en algo tangible y concreto, combinando el concepto explícito recién creado con el conocimiento explícito existente.

Expandir el conocimiento. El nuevo concepto, que ha sido creado, justificado y modelado, continúa adelante hacia un nuevo ciclo de creación de conocimiento en un nivel distinto.

\section{CREACIÓN DE CONOCIMIENTO ORGANIZACIONAL Y LOS MODELOS DE GESTIÓN DE CALIDAD Y DE CONTROL INTERNO}

El Modelo Estándar de Control Interno para el Sector Público y la Norma Técnica de Calidad en la Gestión Pública contienen aspectos comunes que en su implementación coordinada buscan ayudar a las entidades al cumplimiento de sus objetivos y a la búsqueda de la satisfacción de las necesidades y expectativas de las partes interesadas; por esta razón la creación de conocimiento organizacional puede constituirse en una estrategia de desarrollo, mejoramiento y sostenibilidad capaz de impulsar la innovación al interior de las entidades públicas colombianas.

Una vez aclarados los conceptos frente a la creación del conocimiento organizacional, se determinan factores primarios que permiten establecer la articulación de un modelo oriental con uno occidental. Por lo cual, se revisan coincidencias de intención entre las fases de creación del conocimiento organizacional planteada por Naonaka y Takeuchi (1999, pp. 14-15) y los principios, requisitos y elementos de las normas MECI 1000:2014 y NTCGP 1000:2009, tales como:

- Requieren el compromiso, liderazgo y participación activa de la alta dirección y de los servidores públicos. En este aspecto los autores Nonaka y Takeuchi establecen la existencia de jugadores clave en la creación del conocimiento organizacional a los que denominan rangos altos, rangos medios y empleados de primera línea.

- Definen el talento humano como recurso fundamental de la organización. Frente al talento 
humano los autores exaltan el conocimiento tácito existente en los individuos. A este conocimiento se le dan dos dimensiones: 1) Dimensión técnica: relacionada con las habilidades no formales (saber llevar a cabo una tarea) y 2) Dimensión cognoscitiva: asociada a la forma como se percibe el mundo (esquemas, modelos mentales, percepciones); de igual forma la norma de calidad refiere que los servidores públicos deben ser competentes con base en la educación, formación, habilidades y experiencia.

- Conciben la información como un medio para construir conocimiento y mejora continua. Al respecto los autores Nonaka y Takeuchi (1999, p. 63) sostienen que la información tiene dos formas de considerarse: la primera por su volumen y la segunda por su significado; en este sentido, el SGG y MECI poseen un enfoque basado en datos y hechos para la toma de decisiones sin descartar la intuición.
- Entienden a las organizaciones como sistemas abiertos que interactúan con el entorno y que se ven sometidas a continuos cambios dados por los requisitos del mismo (requisitos de partes interesadas). De acuerdo con la intención de la norma NTCGP1000 en su apartado de planificación, el SGC debe estar en capacidad de conservar su integridad frente a los cambios organizacionales, es por eso que la creación del conocimiento organizacional podría convertirse en el mecanismo por el cual las entidades gestionen dichos cambios.

Teniendo claro las coincidencias, para facilitar la articulación de los principios, requisitos y elementos que rigen el SGC y MECI con las fases de la creación del conocimiento organizacional, se determinan unas preguntas orientadoras en la tabla 2 que establecen el relacionamiento enunciando los aspectos que son determinantes para la incorporación de nuevos conceptos, teniendo una visión global de la entidad y su sistema, sin llegar a la particularidad de los cumplimientos de otros requisitos.

Tabla 2. Relacionamiento entre las fases de la Teoría de Creación del Conocimiento y los modelos de gestión de las entidades del Estado.

\begin{tabular}{|l|l|}
\hline \multicolumn{1}{|c|}{$\begin{array}{c}\text { FASE DE LA TEORÍA DE CREACIÓN } \\
\text { DEL CONOCIMIENTO }\end{array}$} & \multicolumn{1}{c|}{ PREGUNTAS ORIENTADORAS } \\
\hline Compartir el conocimiento tácito & $\begin{array}{l}\text { ¿Qué requisitos, elementos y principios presentes en los SGC y MECI propician y } \\
\text { regulan la socialización? }\end{array}$ \\
\hline Crear conceptos & $\begin{array}{l}\text { ¿Qué requisitos, elementos y principios contienen lineamientos que permiten alinear el } \\
\text { conocimiento a los SGC y MECI previamente implementados? }\end{array}$ \\
\hline Justificar los conceptos & $\begin{array}{l}\text { ¿Qué requisitos, elementos y principios de los SGC y MECI contienen lineamientos que } \\
\text { se deberín tener en cuenta para realizar la verificación de los conceptos creados? }\end{array}$ \\
\hline Construir un arquetipo & $\begin{array}{l}\text { ¿Qué requisitos, elementos y principios de los SGC y MECI regulan y contienen } \\
\text { lineamientos susceptibles de modificación a partir de los conceptos creados? }\end{array}$ \\
\hline Expandir el conocimiento & $\begin{array}{l}\text { ¿Qué requisitos, elementos y principios de los SGC y MECI fomentan la mejora continua } \\
\text { respecto a los conceptos creados? }\end{array}$ \\
\hline
\end{tabular}

Fuente: elaboración propia. 
Sin embargo, el ejercicio no busca desviar, modificar o sustituir lo señalado en dichos requisitos y elementos, sino que suministra elementos básicos a tener en cuenta al momento de incorporar nuevos conceptos a los mencionados modelos de gestión. Por lo tanto, a continuación se presentan los esquemas definidos para cada una de las fases, así:

\section{Favorezca la socialización - comparta el conoci-} miento: la fase se basa en compartir experiencias y por tanto conocimiento tácito, el cual presenta comportamientos cíclicos en las organizaciones. Por lo tanto, se identifican los principios, requisitos y elementos pertenecientes a los modelos de gestión de calidad y de control interno, que por su intención y particularidades propicien o favorezcan la socialización; es decir que a través de la existencia de dichas características la organización o entidad pública cuente con el ambiente adecuado para la promoción del diálogo.

Tabla 3. Principios, requisitos y elementos que propician y regulan la generación del caos creativo (fase de socialización).

\begin{tabular}{|c|c|c|c|}
\hline \multicolumn{2}{|r|}{ NTCGP 1000:2009 } & \multicolumn{2}{|r|}{ MECI 1000:2014 } \\
\hline PRINCIPIOS & REQUISITOS & PRINCIPIOS & ELEMENTOS \\
\hline $\begin{array}{l}\text { - Liderazgo } \\
\text { - Participación activa de } \\
\text { los servidores públicos } \\
\text { y/o particulares que } \\
\text { ejercen funciones } \\
\text { públicas } \\
\text { - Enfoque del sistema } \\
\text { para la gestión } \\
\text { - Mejora continua } \\
\text { - Transparencia }\end{array}$ & $\begin{array}{l}\text { 4.1 Requisitos generales } \\
\text { 5.1 Compromiso de la dirección } \\
\text { 5.4.2 Planificación del sistema de gestión } \\
\quad \text { de la calidad } \\
\text { 5.5.1 Responsabilidad y autoridad } \\
\text { 5.5.2 Representante de la dirección } \\
\text { 5.5.3 Comunicación interna } \\
\text { 6.1 Provisión de recursos } \\
\text { 6.2 Talento humano } \\
\text { 6.3 Infraestructura } \\
\text { 6.4 Ambiente de trabajo }\end{array}$ & Autocontrol & $\begin{array}{l}\text { 3. Eje transversal: información y comunicación } \\
\text { 1.1.1 Acuerdos, compromisos y protocolos éticos } \\
\text { 1.1.2 Desarrollo del talento humano } \\
\text { 1.2.2 Modelo de operación por procesos } \\
\text { 1.2.3 Estructura organizacional } \\
\text { 1.3.1 Políticas de administración del riesgo }\end{array}$ \\
\hline
\end{tabular}

Fuente: elaboración propia.

2. Genere el conocimiento: la fase se basa en que el conocimiento se hace tangible por medio del diálogo 0 la reflexión colectiva. En este proceso el conocimiento individual (tácito) se verbaliza y se estructura en conceptos que posteriormente pueden llegar a ser incorporados en la organización en forma de procesos, procedimientos, instrucciones de trabajo, productos y servicios, entre otros. Ahora bien, así como existen principios, requisitos y elementos que favorecen la socialización, es posible pensar en la existencia de otros que por su intención contienen lineamientos o especificaciones que deban ser tenidas en cuenta por parte de los responsables de crear los nuevos conceptos, ya sea porque se requieran para accionar el conocimiento o porque requieran ajustes producto de la generación de los nuevos conceptos. 
Tabla 4: Principios, requisitos y elementos que contienen lineamientos que permiten alinear el conocimiento (fase de exteriorización).

\begin{tabular}{|c|c|c|c|}
\hline \multicolumn{2}{|c|}{ NTCGP 1000:2009 } & \multicolumn{2}{|c|}{ MECI 1000:2014 } \\
\hline PRINCIPIOS & REQUISITOS & PRINCIPIOS & ELEMENTOS \\
\hline $\begin{array}{l}\text { - Enfoque hacia al cliente } \\
\text { - Enfoque basado en } \\
\text { procesos } \\
\text { - Enfoque del sistema para } \\
\text { la gestión }\end{array}$ & $\begin{array}{l}\text { 5.2 Enfoque al cliente } \\
\text { 5.3 Políica de calidad } \\
\text { 5.4.1 Objetivos de calidad } \\
\text { 7.1 Planificación de la realización del } \\
\text { producto o prestación del servicio } \\
\text { 7.2.1 Determinación de los requisitos } \\
\text { relacionados con el producto y/o } \\
\text { servicio } \\
\text { 7.3.1 Planificación del diseño y desarrollo } \\
\text { 7.3.2 Elementos de entrada para el diseño } \\
\text { y desarrollo } \\
\text { 7.5.4 Propiedad del cliente }\end{array}$ & $\begin{array}{l}\text { - Autorregulación } \\
\text { - Autogestión }\end{array}$ & $\begin{array}{l}\text { 1.2.1 Planes, programas y proyectos } \\
\text { 1.2.5 Políticas de operación }\end{array}$ \\
\hline
\end{tabular}

Fuente: elaboración propia.

3. Sustente los conceptos creados: se asociarán los requisitos del SGC y MECI que contienen los lineamientos institucionales que se deben tener en cuenta para realizar la verificación de los nuevos conceptos creados con el fin de garantizar la integridad de los mismos.

Tabla 5. Principios, requisitos y elementos que contienen lineamientos que se deben tener en cuenta para realizar la verificación de los conceptos creados (fase de justificar los conceptos).

\begin{tabular}{|c|c|c|c|}
\hline \multicolumn{2}{|r|}{ NTCGP 1000:2009 } & \multicolumn{2}{|c|}{ MECI 1000:2014 } \\
\hline PRINCIPIOS & REQUISITOS & PRINCIPIOS & ELEMENTOS \\
\hline $\begin{array}{l}\cdot \text { Enfoque hacia el cliente } \\
\text { - Enfoque basado en } \\
\text { procesos } \\
\text { - Enfoque del sistema para } \\
\text { la gestión }\end{array}$ & $\begin{array}{l}\text { 7.2.2 Revisión de los requisitos relacionados con el producto y/o } \\
\text { servicio } \\
\text { 7.2.3 Comunicación con el cliente } \\
\text { 7.3.3 Resultados del diseño y desarrollo } \\
\text { 7.3.4 Revisión del diseño y desarrollo } \\
\text { 7.3.5 Verificación del diseño y desarrollo } \\
\text { 7.5.5 Preservación del producto y/o servicio } \\
\text { 7.3.6 Validación del diseño y desarrollo } \\
\text { 7.3.7 Control de cambios del diseño y desarrollo }\end{array}$ & No aplica & No aplica \\
\hline
\end{tabular}

Fuente: elaboración propia.

\section{Actualice el sistema de gestión de calidad y el} sistema de control interno: la fase se fundamenta en la materialización de la inclusión del concepto creado en los lineamientos de la organización para ser difundidos e interiorizados en los niveles que corresponden. Por lo tanto, los requisitos y elementos son aquellos relacionados con la regulación de lineamientos y prácticas de la entidad susceptibles de pasar por una modificación explícita traducida en la estructura documental. 
Tabla 6. Principios, requisitos y elementos que regulan y contienen lineamientos susceptibles de modificación a partir de la creación de los nuevos conceptos (fase de construir el arquetipo).

\begin{tabular}{|c|c|c|c|}
\hline \multicolumn{2}{|r|}{ NTCGP 1000:2009 } & \multicolumn{2}{|c|}{ MECI 1000:2014 } \\
\hline PRINCIPIOS & REQUISITOS & PRINCIPIOS & ELEMENTOS \\
\hline $\begin{array}{l}\text { - Enfoque basado en procesos } \\
\text { - Enfoque basado en hechos } \\
\text { y datos para la toma de } \\
\text { decisiones } \\
\text { - Relaciones mutuamente } \\
\text { beneficiosas con los } \\
\text { proveedores de bienes o } \\
\text { servicios } \\
\text { - Coordinación, cooperación y } \\
\text { articulación }\end{array}$ & $\begin{array}{l}\text { 4.2.1 Generalidades } \\
\text { 4.2.2 Manual de calidad } \\
\text { 4.2.3 Control de documentos } \\
\text { 4.2.4 Control de registros } \\
\text { 7.4.1 Proceso de adquisición de bienes y servicios } \\
\text { 7.4.2 Información para la adquisición de bienes y } \\
\text { servicios } \\
\text { 7.4.3 Verificación de los productos y/o servicios } \\
\text { adquiridos } \\
\text { 7.5.1 Control de la producción y de la prestación del } \\
\text { servicio } \\
\text { 7.5.2 Validación de los procesos de la producción y } \\
\text { de la prestación del servicio }\end{array}$ & Autogestión & $\begin{array}{l}\text { 1.3.2 Identificación del } \\
\text { riesgo } \\
\text { 1.3.3 Análisis y valoración } \\
\text { del riesgo }\end{array}$ \\
\hline
\end{tabular}

Fuente: Elaboración propia.

5. Asegure la mejora continua: la fase se fundamenta en la generación de nuevos ciclos de creación de conocimiento, una vez se ha difundido e incorporado el nuevo concepto en la organización. Por lo tanto, se identifican aquellos requisitos y elementos que fomentan la mejora continua y evolución organizacional respecto a los conceptos creados, basados en el control, medición y evaluación de la información resultante de la aplicación de la espiral de conocimiento.

Tabla 7. Principios, requisitos y elementos que fomentan la mejora continua sobre los conceptos creados (fase de expandir el conocimiento).

\begin{tabular}{|c|c|c|c|}
\hline \multicolumn{2}{|r|}{ NTCGP 1000:2009 } & \multicolumn{2}{|r|}{ MECI 1000:2014 } \\
\hline PRINCIPIOS & REQUISITOS & PRINCIPIOS & ELEMENTOS \\
\hline $\begin{array}{l}\text { - Mejora continua } \\
\text { - Enfoque basado en } \\
\text { hechos y datos para la } \\
\text { toma de decisiones }\end{array}$ & $\begin{array}{l}\text { 5.6 Revisión por la dirección } \\
\text { 7.5.3 Identificación y trazabilidad } \\
\text { 7.6 Control de los equipos de seguimiento y de } \\
\quad \text { medición } \\
\text { 8.1 Generalidades } \\
\text { 8.2.1 Satisfacción del cliente } \\
\text { 8.2.2 Auditoría interna } \\
\text { 8.2.3 Seguimiento y medición de los procesos } \\
\text { 8.2.4 Seguimiento y medición del producto y/o } \\
\quad \text { servicio } \\
\text { 8.3 Control del producto y/o servicio no conforme } \\
\text { 8.4 Análisis de datos } \\
\text { 8.5.1 Mejora continua } \\
\text { 8.5.2 Acción correctiva } \\
\text { 8.5.3 Acción preventiva }\end{array}$ & Autogestión & $\begin{array}{l}\text { 1.2.4 Indicadores de gestión } \\
\text { 2.1.1 Autoevaluación de control y } \\
\text { gestión }\end{array}$ \\
\hline
\end{tabular}

Fuente: elaboración propia. 


\section{VALIDACIÓN DE LA METODOLOGÍA}

La propuesta aquí presentada se validó en la Autoridad de Licencias Ambientales (ANLA), con el objeto de conocimiento "participación ciudadana y control social", donde se identificaron y aplicaron los ajustes al documento en cuanto a claridad, pertinencia y objetividad, determinando el estado de los aspectos de interés definidos en el ejercicio de triangulación para el diseño del instrumento, los cuales se encuentran enfocados a canales de comunicación, procedimientos establecidos para el tratamiento de la información, definición del concepto, flujo de la información y utilidad de la información; lo que generó cinco lemas asociados a los mecanismos de participación ciudadana y control social sobre los cuales se determinaron los nuevos conceptos a incorporar en los sistemas de gestión de la entidad.

A través de esta validación realizada mediante la implementación de la "Propuesta metodológica para incorporar la creación del conocimiento a los modelos de gestión de calidad y estándar de control interno del Estado" en una entidad pública colombiana del orden nacional, fue posible identificar e incorporar nuevos objetos de conocimiento asociados a las premisas de desarrollo administrativo y al logro de los objetivos del estado, aplicando el modelo de Creación del Conocimiento Organizacional planteado por Nonaka y Takeuchi; por lo tanto, los lineamientos de orientación planteados en dicha propuesta se pueden aplicar de forma práctica en cualquier organización que cuente con sistemas de gestión implementados, con el fin de facilitar y guiar la generación e incorporación de nuevos conceptos organizacionales.

\section{CONCLUSIONES}

Como resultado de la investigación se logra relacionar las Fases de la Creación del Conocimiento
Organizacional con los modelos de gestión de calidad y de control interno, ya que aun cuando la teoría formulada por Nonaka y Takeuchi es un modelo oriental de creación del conocimiento organizacional fundamentado en el empirismo, mientras que los sistemas de gestión de calidad y de control interno están basados en modelos occidentales cimentados en el racionalismo, a través de la exploración documental y el análisis de la intencionalidad de dichas herramientas, fue posible identificar un vínculo con los principios, requisitos y elementos del SGC y MECI. Dicho vínculo se fundamenta en que la Teoría de Creación del Conocimiento Organizacional reconoce la existencia de condiciones que facilitan el flujo de conocimiento y que además propone una serie de fases cuyas características son equiparables a la intencionalidad de los principios, requisitos y elementos de los modelos de gestión.

Algunas de las coincidencias encontradas a partir de la exploración documental fueron: 1) Requieren el compromiso, liderazgo y participación activa de la alta dirección y de los miembros de la entidad. 2) Definen el talento humano como recurso fundamental. 3) Conciben la información como un medio para construir conocimiento y mejora continua. 4) Conciben a las organizaciones como sistemas abiertos que interactúan con el entorno y que se ven sometidas a continuos cambios dados por los requisitos del mismo (requisitos de partes interesadas).

En el caso de organizaciones no gubernamentales, se considera que estos lineamientos pueden ser aplicables en aquellas entidades que tengan un sistema de gestión de calidad implementado bajo la norma ISO 9001 y un sistema de control interno basado en el modelo del Commitee of Sponsoring Organizations (Comité de Organizaciones Patrocinadoras), conocido por sus siglas en inglés como COSO, dado que estos dos modelos son los principales referentes de los estándares de calidad y MECI adoptados en el sector público colombiano. 
Es importante resaltar que la propuesta metodológica recopila la formulación de una serie de lineamientos basados en las reflexiones planteadas en la obra de Nonaka y Takeuchi, que son complementarias a los modelos de gestión de calidad y de control interno, y que además incorporan terminología y aspectos a tener en cuenta por las entidades del Estado del nivel nacional, interesadas en generar y adherir nuevos conceptos a la gestión institucional y garantizando el cumplimiento de las temáticas establecidas en las políticas de desarrollo administrativo establecidas por ley. Uno de los términos mayormente utilizado son los denominados "objetos de conocimiento", cuya intención es enfocar el ejercicio de producción de conocimiento organizacional.

Dentro de los lineamientos identificados y referenciados en la propuesta metodológica se encuentran: 1) La incorporación de estrategias de fomento al desarrollo de conocimiento grupal. 2) La forma de participación de los rangos directivo, medio y de primera línea en los procesos de creación de conocimiento organizacional. 3) La asignación de recursos presupuestales, físicos y tecnológicos para el desarrollo del nuevo conocimiento. 4) El establecimiento de responsabilidades frente a la creación del conocimiento y la definición de canales de comunicación entre los diferentes rangos de autoridad en la entidad. 5) La identificación y ajuste del sistema de gestión de calidad y del sistema de control interno a partir de la incorporación de objetos de conocimiento. 6) La incorporación de la temporalidad de la creación del conocimiento respecto a la formulación de planes de acción y presupuesto institucional.

La propuesta metodológica pudo ser validada a través del ejercicio de simulación realizado en la ANLA, en donde a partir de la identificación y conformación de equipos de trabajo se lograron ajustar y ratificar aspectos de contenidos y forma en la propuesta metodológica. De este ejercicio se concluye que: 1) Es posible incorporar objetos de conocimiento a los modelos de gestión de calidad y control interno utilizando las fases de creación del conocimiento expuestas por Nonaka y Takeuchi. 2) Tal como lo exponen los autores de la Teoría de Creación del Conocimiento, el uso de lemas fomenta la creatividad en los grupos de trabajo y permite hacer explícito el conocimiento tácito. 3) Por su intención, los principios, requisitos y elementos juegan un papel diferenciador dentro de las fases de la Teoría de Creación del Conocimiento. Este papel se encuentra dado por su intención orientadora, reguladora, de verificación o de mejora continua. 4) A través de los principios, requisitos y elementos de los modelos de gestión de calidad y de control interno es posible operacionalizar la Teoría de Creación del Conocimiento, lo anterior, en beneficio de la mejora continua de las entidades.

\section{REFERENCIAS}

Cisterna, F. (2005). Categorización y triangulación como procesos de validación del conocimiento en investigación cualitativa. Theoria, 14(1), 68. Recuperado de https://www.redalyc.org/articulo. oa?id=29900107

Congreso de la República. (1998, 29 de diciembre). Ley 489. Por la cual se dictan normas sobre la organización y funcionamiento de las entidades del orden nacional, se expiden las disposiciones, principios y reglas generales para el ejercicio de las atribuciones previstas en los numerales 15 y 16 del artículo 189 de la Constitución Política y se dictan otras disposiciones. Diario Oficial 43464. Bogotá, Colombia.

Departamento Administrativo de la Función Pública. (2014). Manual técnico del modelo estándar de control interno para el estado colombiano - MECI. 
Hernández, R., Fernández, C., y Baptista, P. (2010). Peluffo, M. B., y Catalán, E. (2002, diciembre). IntroMetodología de la investigación. Bogotá D. C.: ducción a la gestión del conocimiento y su apliMcGraw-Hill. cación en el sector público. Serie Manuales, (22). Santiago de Chile: Instituto Latinoamericano y

Instituto Colombiano de Normas Técnicas y Certificación - Icontec. (2009). Norma Técnica de Calidad en la Gestión Pública. NTCGP 1000:2009. Bogotá D. C.: Icontec.

Nonaka, I., y Takeuchi, H. (1999). La organización creadora de conocimiento, Cómo las compañías japonesas crean la dinámica de la innovación. México D. F.: Oxford University Press. del Caribe de Planificación Económica y Social IPLES. Naciones Unidas, CEPAL, ECLAC.

Presidencia de la República. (2005, 20 de mayo). Decreto 1599. Por el cual se adopta el Modelo Estándar de Control Interno. Diario Oficial 45920. Bogotá, Colombia. . (2009, 18 de noviembre). Decreto 4485. Por medio de la cual se adopta la actualización de la Norma Técnica de Calidad en la Gestión Pública. Diario Oficial 47538. Bogotá, Colombia. 